\title{
Activation of astrocytes and expression of inflammatory cytokines in rats with experimental autoimmune encephalomyelitis
}

\author{
LIZHI ZHANG ${ }^{1}$, HUALEI XIE ${ }^{2}$ and LIHAI CUI ${ }^{3}$ \\ ${ }^{1}$ Department of Internal Neurology, Daqing Longnan Hospital, Daqing, Heilongjiang 163001; \\ Departments of ${ }^{2}$ Emergency and ${ }^{3}$ Neurology, The Second People's Hospital of Liaocheng, \\ Liaocheng, Shandong 252601, P.R. China
}

Received January 3, 2018; Accepted July 17, 2018

DOI: 10.3892/etm.2018.6798

\begin{abstract}
The aim of the study was to investigate and discuss the activation of astrocytes and the expression of inflammatory cytokines in rats with experimental autoimmune encephalomyelitis (EAE). Twenty Wistar rats were randomly divided into the normal control $(n=10)$ and EAE group $(n=10)$. The rats in the EAE group were injected intraperitoneally with myelin oligodendrocyte glycoprotein 35-55 emulsion, and those in the control group were injected with the equivalent volume of normal saline. Wear neurological function scale was applied to evaluate the neurological functions of the rats, and the weight changes were recorded. At 21 days after immunization, hematoxylin and eosin staining was used to detect the histomorphology, and immunofluorescence was used to measure the activation conditions of the brain astrocytes. Reverse transcription-polymerase chain reaction and western blot analysis were utilized to detect the messenger RNA (mRNA) and protein levels of inflammatory factors. The disease occurred in rats of the EAE group at 9 days after immunization, and the incidence rate was $80 \%$. The Wear score of the rats in the EAE group was significantly increased compared with that in the control group $(\mathrm{P}<0.05)$. At 9 days after immunization, the weight of the rats in the EAE group was obviously lower than that in the control group $(\mathrm{P}<0.05)$. The inflammatory lesion of rats in the EAE group mainly occurred in the region of brain parenchyma. The glial fibrillary acidic protein level in the brain sections of the rats in the EAE group was markedly elevated compared with that in control group. The mRNA and protein levels of interleukin-10 in the rat brain in EAE group were decreased notably $(\mathrm{P}<0.05)$, while those of interferon- $\gamma$ and
\end{abstract}

Correspondence to: Dr Lihai Cui, Department of Neurology, The Second People's Hospital of Liaocheng, 306 Jiankang Road, Liaocheng, Shandong 252601, P.R. China

E-mail: zaiyan796306@163.com

Key words: inflammatory factors; experimental autoimmune encephalomyelitis; astrocytes tumor necrosis factor- $\alpha$ were increased significantly $(\mathrm{P}<0.05)$. The significant increases in the activation level of astrocytes and inflammatory cytokine level have a close relationship with EAE progression.

\section{Introduction}

Experimental autoimmune encephalomyelitis (EAE) is a kind of autoimmune disease mediated by cluster of differentiation 4+ $\mathrm{T}$ cell (1), whose characteristic is that mononuclear cell infiltration occurs in the central nervous system (CNS) and around the small vessels, while inflammation is the vital cause for the occurrence and development of $\operatorname{EAE}(2,3)$. The pathogenesis of the disease is very complicated and is associated with the activation of immune cells, destruction of blood-brain barrier and activation of glial cells (4).

Astrocytes are the satellite cells of neurons and a category of glial cells with the largest volume (5). Many long and branched processes generated from the body of astrocyte extend and fill up the space between the bodies of nerve cells and their processes, playing a part in supporting and separating the nerve cells (6). In addition, astrocytes can exert neuroprotective effects through the mechanisms of downregulating the inflammatory responses of the CNS and alleviating the oxidative stress (7). Neuroglia cells participate in the pathophysiological processes of inflammations; for instance, the activation of microglia is an important participant of removing necrotic debris and other foreign substances (8). However, the hyperactivation of astrocytes in the EAE models can accelerate the activation of inflammatory transcription factors and multiple inflammatory mediators and initiate inflammatory cascades, thus promoting the progression of disease (9).

This study aimed to analyze the activation conditions of the astrocytes in the EAE models and the association of inflammatory factor levels expressed by them with the disease.

\section{Materials and methods}

Laboratory animals and EAE modeling. Wistar rats (weighing $200 \pm 18 \mathrm{~g}$, male) were purchased from and fed at the Jiangsu 
Table I Primer sequences for RT-qPCR.

\begin{tabular}{|c|c|c|}
\hline Gene name & Primer sequence & Length (bp) \\
\hline \multirow[t]{2}{*}{$I L-10$} & 5'-GAGATCTCCGAGATGCCTTCAG-3' & 248 \\
\hline & 5'-CAAGGACTCCTTTAACAACAAGTTGT-3' & \\
\hline \multirow[t]{2}{*}{$T N F-\alpha$} & 5'-CAGGGGCCACCACGCTCTTC-3' & 189 \\
\hline & 5'-CTTGGGGCAGGGGCTCTTGAC-3' & \\
\hline \multirow[t]{2}{*}{$I F N-\gamma$} & 5'-GCTGTCATAATAATATTCAGAC-3' & 273 \\
\hline & 5'-CGAGCTTTAAAAGATAGTTCC-3' & \\
\hline \multirow[t]{2}{*}{$G A P D H$} & 5'-AGGTCGGTGTGAACGGATTTG-3' & 254 \\
\hline & 5'-TGTAGACCATGTAGTTGAGGTCA-3' & \\
\hline
\end{tabular}

IL-10, interleukin-10; TNF- $\alpha$, tumor necrosis factor- $\alpha$; IFN- $\gamma$, interferon- $\gamma$; GAPDH, glyceraldehyde-3-phosphate dehydrogenase. RT-qPCR, reverse transcription polymerase chain reaction.

Laboratory Animal Center. The rats were fed in a specific pathogen-free environment at a room temperature of $25 \pm 2^{\circ} \mathrm{C}$, with free intake of food and water. The study was approved by the Ethics Committee of Daqing Longnan Hospital (Daqing, China).

The EAE models were established according to foreign literature (10): Myelin oligodendrocyte glycoprotein 35-55 (MOG35-55) (article number: 2568, R\&D Systems Europe, Ltd., Abingdon, UK, $400 \mu \mathrm{g}$ ), mycobacterium tuberculosis H37Ra (400 $\mu \mathrm{g})$, phosphate-buffered saline (PBS) $200 \mu \mathrm{l}$; and complete Freund's adjuvant (CFA) solution $100 \mu \mathrm{l}$ were mixed; and the mixture was made into milky-white suspension through ultrasonic emulsification. The rats in the EAE group were intraperitoneally injected with immunopotentiator pertussis toxin $(500 \mathrm{ng} / \mathrm{rat})$ at 0 and 2 days, followed by $0.1 \mathrm{ml}$ MOG35-55 emulsion. Rats in the control group were injected with an equivalent volume of normal saline. The clinical symptoms of the rats were observed for 20 days, and scores were recorded in accordance with the Wear neurological function scale as: 0 point, no signs; 1 , limp tail and ataxia; 3 , paralysis of one hind limb; 4, paralysis of both hind limbs; and 5 , dying or death.

Hematoxylin and eosin $(H \& E)$ staining. The rats were anaesthetised with pentobarbital $(2.5 \mathrm{mg} / 100 \mathrm{~g})$ then sacrificed by decapitation. The brains of the two groups of rats were fixed in an appropriate amount of formalin overnight, which were then dehydrated in graded alcohol, cleared, wax-impregnated and embedded in paraffin, after which the paraffin tissues were sliced to $0.4 \mu \mathrm{m}$. The sections were baked in an oven at $65^{\circ} \mathrm{C}$ for 3-5 h, followed by dewaxing in xylene reagent and rehydration with graded ethanol. H\&E staining, rehydration with graded ethanol and drying of sections were conducted in sequence, and the sections were mounted with neutral balsam. The histomorphology of the sections was observed and photographed under a microscope (DM-5000B, Leica Microsystems, Wetzlar, Germany).

Detection of activation conditions of astrocytes via immunofluorescence. The brain tissue sections of the rats were permeabilized with $0.1 \%$ Triton X-100 for 10 min after dewaxing and rehydration with ethanol, which were then blocked in 5\% standard protein bovine serum albumin for $30 \mathrm{~min}$. The rabbit anti-rat primary antibodies to glial fibrillary acidic protein (GFAP) polyclonal antibody were added to the sections in drops and blocked at $4^{\circ} \mathrm{C}$ overnight (1:200; cat. no. ab7260; Abcam, Cambridge, UK). The sections were washed in PBS with Tween-20 three times the next day, followed by incubation with Alexa Fluor-labeled fluorescent goat anti-rabbit secondary polyclonal antibody (1:1,000; cat. no. A-11034; Thermo Fisher Scientific, Inc., Waltham, MA, USA) at room temperature for $1 \mathrm{~h}$. The sections were mounted after 4', 6-diamidino-2-phenylindole was added for visualization of cell nuclei for $3 \mathrm{~min}$, which were observed and photographed under an inverted fluorescence microscope (DM-6000B, Leica Microsystems).

Reverse transcription-quantitative polymerase chain reaction (RT-qPCR). TRIzol reagent (Invitrogen; Thermo Fisher Scientific, Inc.) was applied to extract the total RNA in the brain tissue of the rats. The RNA concentration was detected using a spectrophotometer (Bio-Rad Laboratories, Inc., Hercules, CA, USA). RNA (1 $\mu \mathrm{g})$ was withdrawn for reverse transcription (Takara Bio, Inc., Otsu, Japan), and the obtained complementary DNA was stored at $-20^{\circ} \mathrm{C}$. The messenger RNA (mRNA) levels of various indexes were measured in accordance with the instructions of an All-in-One ${ }^{\mathrm{TM}}$ qPCR Mix (GeneCopoeia, Inc., Rockville, MD, USA) kit. Reaction conditions of RT-qPCR were: Step 1 , at $90^{\circ} \mathrm{C}$ for $10 \mathrm{~min}$; 2, at $95^{\circ} \mathrm{C}$ for $10 \mathrm{sec} ; 3$, at $60^{\circ} \mathrm{C}$ for $20 \mathrm{sec}$ and 4 , at $70^{\circ} \mathrm{C}$ for $20 \mathrm{sec}$; 45 cycles in total. Glyceraldehyde-3-phosphate dehydrogenase (GAPDH) was selected as the internal reference. The formula for the mRNA relative expression levels of the indexes was: $2^{-\Delta \Delta \mathrm{Cq}}[\Delta \mathrm{Cq}=\mathrm{Cq}$ (target gene) $-\mathrm{Cq}(\mathrm{GAPDH})]$. The corresponding primer sequences are shown in Table I.

Western blot analysis. Brain tissues $(5 \mathrm{~g})$ of the rats were cut up and placed into a $2.5 \mathrm{ml}$ Eppendorf tube, to which $150 \mu \mathrm{l}$ mixture of radio immtmoprecipitation assay lysis buffer and protease inhibitor Cocktail (Sigma-Aldrich; Merck KGaA, Darmstadt, Germany) was added, followed by homogenizing in a tissue homogenizer for $10 \mathrm{~min}$. After centrifugation at $12,000 \mathrm{x} \mathrm{g}$ for $15 \mathrm{~min}\left(\right.$ at $\left.4^{\circ} \mathrm{C}\right)$, the upper liquid, namely, tissue protein, was absorbed. The concentration of the protein was measured using a bicinchoninic acid protein assay kit 


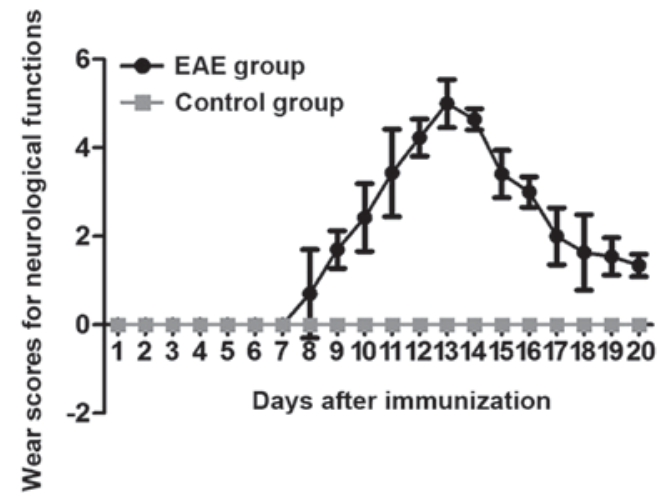

Figure 1. Wear scores for neurological functions of the two groups of rats.

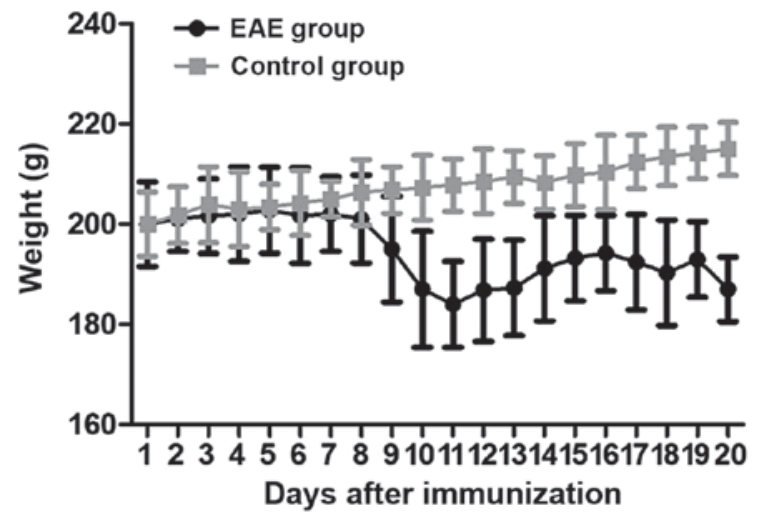

Figure 2. Weight changes of the two groups of rats.

(Beyotime Institute of Biotechnology, Haimen, China). After being denatured, the total protein was separated by $8 \%$ sodium dodecyl sulfate-polyacrylamide gel electrophoresis, which was transferred to the nitrocellulose membrane (Merck KGaA). The bands were blocked in 5\% skim milk for $1 \mathrm{~h}$ and then incubated in monoclonal antibodies overnight. Interleukin-10 (IL-10; Abcam, 1:1,000), tumor necrosis factor- $\alpha$ (TNF- $\alpha$;
Abcam, 1:2,000) and interferon- $\gamma$ (IFN- $\gamma$; Abcam, 1:1,000) were added. The bands were then incubated in secondary antibodies of anti-rabbit immunoglobulin $\mathrm{G}$ for $1 \mathrm{~h}$ after the membrane was washed, and enhanced chemiluminescence system (Bio-Rad Laboratories, Inc.) was used to reveal the bands of target proteins.

Statistical analysis. GraphPad Prism software (Version 5.01; GraphPad Software, Inc., La Jolla, CA, USA) was utilized for analysis. Independent-sample t-test was applied to compare the differences in the indexes between the two groups of samples. $\mathrm{P}<0.05$ was considered to indicate a statistically significant difference.

\section{Results}

Wear scores for neurological functions of rats. There was no onset of disease in rats of the control group from beginning to end. However, in the EAE group, the disease occurred in the 10 rats at 9 days after immunization, with an average onset time of $5.4 \pm 1.5$ days. Eight rats were attacked by the disease, 1 rat was not attacked and 1 rat was dead; the incidence rate was $80 \%$. During the onset of disease, the Wear score of the rats in EAE group was markedly increased $(\mathrm{P}<0.05)$ (Fig. 1). In addition, the weight of the rats in EAE group at 9 days after immunity was markedly lower than that in the control group $(\mathrm{P}<0.05)$ (Fig. 2).

Detection of brain histomorphology of rats via HE staining. As shown in Fig. 3, the brain tissue sections of the control and EAE groups were compared, and it was indicated that the inflammatory lesion was mainly located in the region of brain parenchyma in rats of the EAE group, which was presented as local infiltration of inflammatory cells dominated by lymphocytes and monocytes. It confirmed that the EAE model had been successfully established.

Detection of activation conditions of astrocytes via immunofluorescence. The activation degree of the astrocytes in the

\section{Control group}

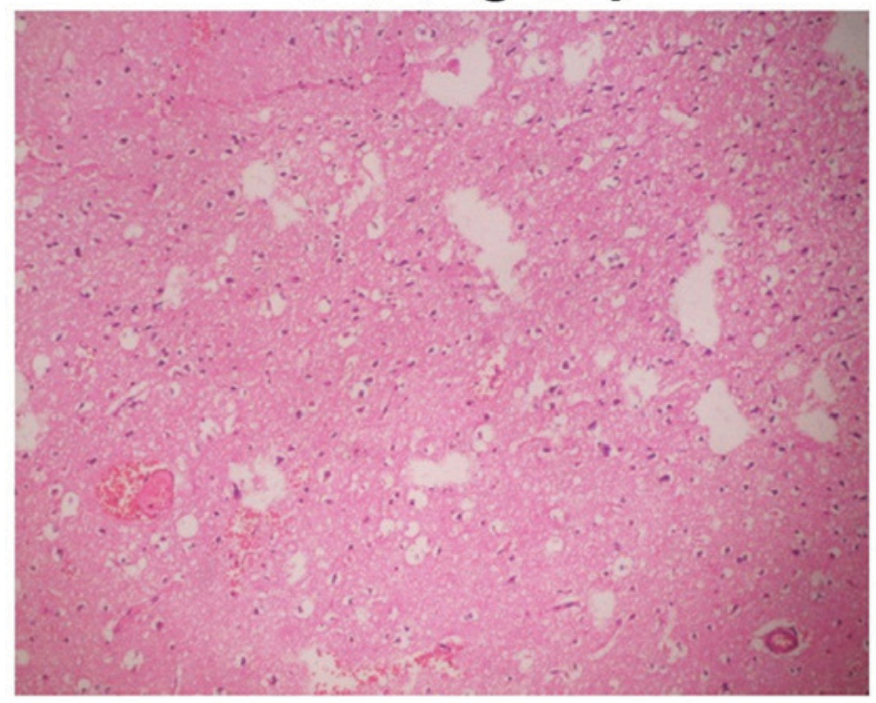

\section{EAE group}

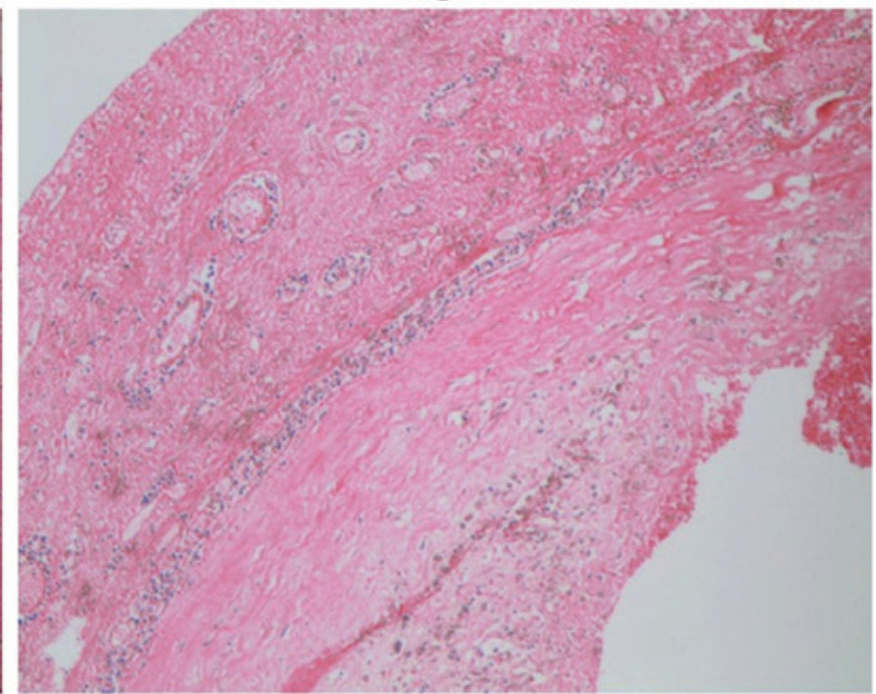

Figure 3. Examination of histopathology of the two groups of rats via H\&E staining. H\&E, hematoxylin and eosin. 

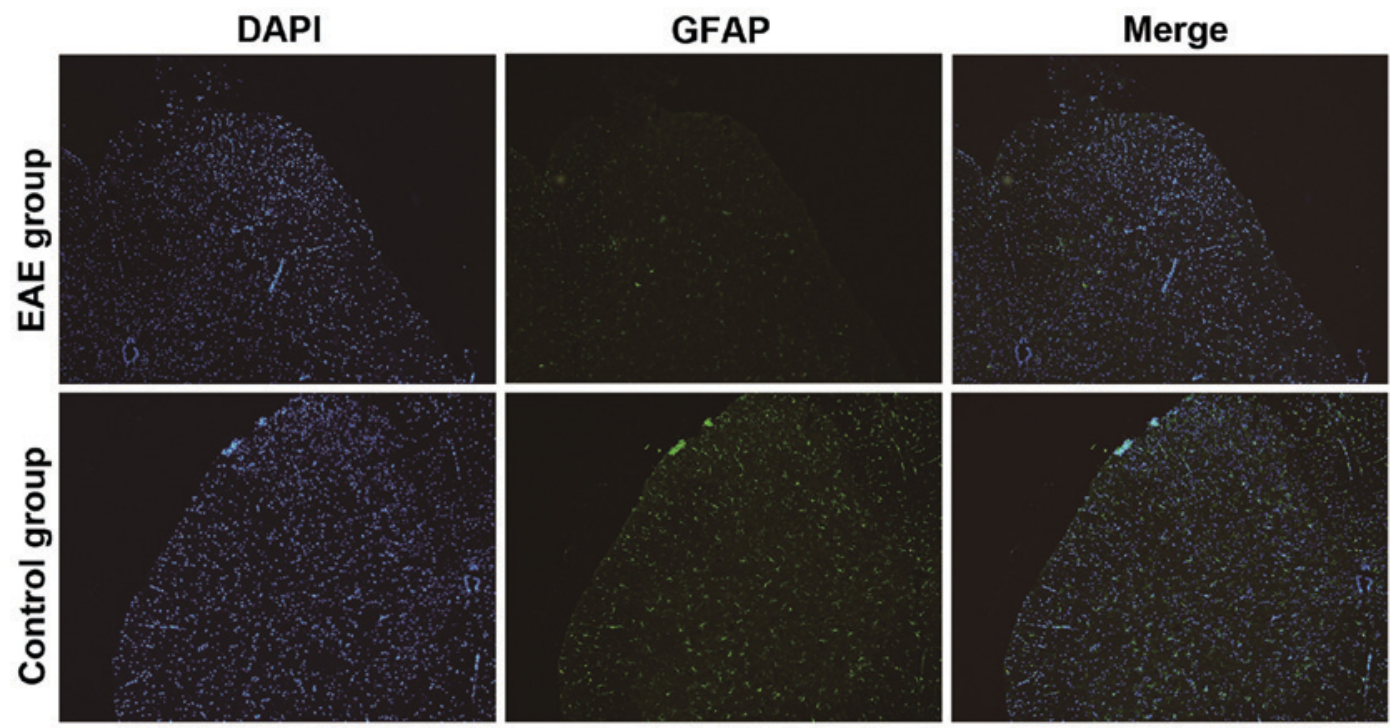

Figure 4. Detection of activation conditions of astrocytes in two groups of rat brains. EAE, experimental autoimmune encephalomyelitis; DAPI, 4', 6-diamidino-2-phenylindole; GFAP, glial fibrillary acidic protein.

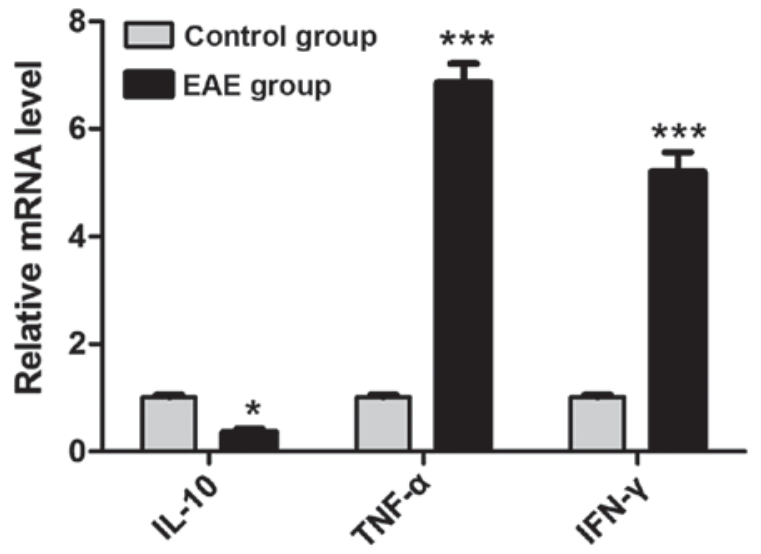

Figure 5. Detection of expression levels of inflammatory factors in two groups of rat brains via RT-qPCR. Compared with that in control group, ${ }^{*} \mathrm{P}<0.05 ;{ }^{* * *} \mathrm{P}<0.001$. RT-qPCR, reverse transcription-quantitative polymerase chain reaction; IL-10, interleukin-10; TNF- $\alpha$, tumor necrosis factor- $\alpha$; IFN- $\gamma$, interferon- $\gamma$.

rat CNS was detected using GFAP specificity. In Fig. 4, the green fluorescence intensity represented the expression level of GFAP. The GFAP level in the brain sections of rats in EAE group was notably elevated compared with that in control group, suggesting that the activation degree of the astrocytes was significantly increased in the EAE rat models.

Detection of inflammatory cytokine expressions via RT-qPCR. As shown in Fig. 5, compared with those in control group, the mRNA expression level of IL-10 in the brains of the rats in EAE group was lowered obviously $(\mathrm{P}<0.05)$, while the mRNA expression levels of IFN- $\gamma$ and TNF- $\alpha$ were elevated notably $(\mathrm{P}<0.05)$.

Detection of inflammatory cytokine expressions via western blot analysis. In order to further investigate the expression of inflammatory factors in the EAE models, western blot analysis was performed to measure the levels of inflammatory factors

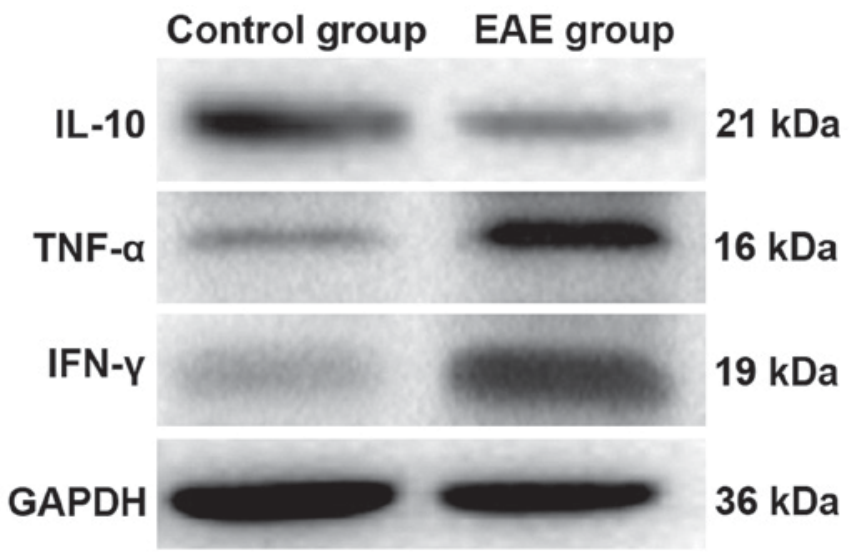

Figure 6. Detection of expression levels of inflammatory factors in two groups of rat brains via western blot analysis. IL-10, interleukin-10; TNF- $\alpha$, tumor necrosis factor- $\alpha$; IFN- $\gamma$, interferon- $\gamma$; GAPDH, glyceraldehyde-3-phosphate dehydrogenase.

in the brain tissues of the two groups of rats. Fig. 6 shows that, the protein level of IL-10 in the brains of rats in the EAE group was decreased significantly $(\mathrm{P}<0.05)$, while those of IFN- $\gamma$ and TNF- $\alpha$ were markedly increased $(\mathrm{P}<0.05)$.

\section{Discussion}

The pathogenesis of EAE is very complex, and the T helper cell type 1 (Th1)/Th2 imbalance which has been identified currently is the main feature of the EAE pathological process (11), in which Th1 plays a major role. As a group of small molecular polypeptides regulating the immune responses, cytokines play vital parts in the regulation of the immune system (12). The encephalitogenic cytokine IFN- $\gamma$ and TNF- $\alpha$ which are associated with inflammatory responses mediated by Th1 can promote the activation and infiltration of $\mathrm{T}$ cells and macrophages and directly damage the blood-cerebrospinal fluid barrier and myelin sheaths 
surrounding the neurons, further expanding the inflammatory lesion $(13,14)$. TNF- $\alpha$ is mainly secreted by the monocytes in the CNS, most of which have been identified as phenotype cells of microglia and macrophages. Inducing the proliferation of astrocytes can increase the severity of EAE (15).

IL-10 is a type of cytokine secreted by Th2 and has the immunosuppressive function; it can inhibit the Th1-mediated immune responses (16). Furthermore, Ouyang et al (17) revealed that IL-10 also has an anti-inflammatory effect. Specifically, IL-10 may influence the pathogenesis of EAE at the initiating stages of the $\mathrm{T}$ cell, including recruitment of inflammatory cells for the CNS and destruction of the CNS tissues. IL-10 can significantly strengthen these cells to suppress the inflammatory lesion of the CNS and improve the capability of autoimmune reaction, thus further decreasing the damage to the myelin sheaths $(17,18)$. Furthermore, IL-10 can enhance the differentiation of transplanted neural stem cells into neurons and lower the possibility of astrocyte hyperplasia at the same time. It can also accelerate the growth of myelin sheaths and axons, which is also an important factor for the pathogenesis of EAE (19).

Previous findings have indicated that the action of astrocytes is involved in the EAE progression (20). Therefore, in this research, the EAE rat models were successfully established by injecting MOG33-35 into the spinal cord, which was consistent with the report of Wang et al (21); EAE rats had loss of myelin sheaths and infiltration of inflammatory cells in the brain; the weight changes were recorded at the same time; the disease occurred in the rats in EAE group one after another at 10 days after immunization, of which the clinical manifestations and Wear scores met the modeling standards. According to the report of Wang et al (21), the brain tissues of the rats were fixed at 21 days after immunization. Since GFAP is the skeleton protein of the astrocytes, its expression level can reflect the degrees of astrocyte hyperplasia and necrosis; the activation conditions of the astrocytes in the rat brain tissues was determined by means of immunofluorescence in this research, and the expression levels of the inflammatory factors in the brain tissue cells of the two groups of rats were detected simultaneously. It was found that the number of activated astrocytes in the brains of EAE rat models was increased obviously; furthermore, RT-qPCR and western blot analysis experiments suggested that the level of anti-inflammatory cytokine IL-10 which was expressed by the astrocytes of EAE rats was markedly decreased, while that of inflammatory factors TNF- $\alpha$ and IFN $-\gamma$ was elevated significantly.

In conclusion, the results of the present study are that, the expression of inflammatory factors IFN- $\gamma$ and TNF- $\alpha$ were markedly increased, while the expression of anti-inflammatory cytokine IL-10 was decreased during the development of EAE, accompanied with the significant activation of astrocytes at the same time. It is suggested that astrocytes and inflammatory factors (IL-10, TNF- $\alpha$ and IFN- $\gamma$ ) play crucial roles in the occurrence and development of EAE.

\section{Acknowledgements}

Not applicable.

\section{Funding}

No funding was received.

\section{Availability of data and materials}

The datasets used and/or analyzed during the current study are available from the corresponding author on reasonable request.

\section{Authors' contributions}

LZ and HX were responsible for immunofluorescence. $\mathrm{LZ}$ and LC helped with PCR and western blot analysis. All authors read and approved the final manuscript.

\section{Ethics approval and consent to participate}

The study was approved by the Ethics Committee of Daqing Longnan Hospital (Daqing, China).

\section{Patient consent for publication}

Not applicable.

\section{Competing interests}

The authors declare that they have no competing interests.

\section{References}

1. Kumar V, Stellrecht K and Sercarz E: Inactivation of T cell receptor peptide-specific $\mathrm{CD} 4$ regulatory $\mathrm{T}$ cells induces chronic experimental autoimmune encephalomyelitis (EAE). J Exp Med 184: 1609-1617, 1996.

2. Reboldi A, Coisne C, Baumjohann D, Benvenuto F, Bottinelli D, Lira S, Uccelli A, Lanzavecchia A, Engelhardt B and Sallusto F: $\mathrm{C}$-C chemokine receptor 6-regulated entry of $\mathrm{T}_{\mathrm{H}}-17$ cells into the CNS through the choroid plexus is required for the initiation of EAE. Nat Immunol 10: 514-523, 2009.

3. Kroenke MA, Carlson TJ, Andjelkovic AV and Segal BM: IL-12-and IL-23-modulated T cells induce distinct types of EAE based on histology, CNS chemokine profile, and response to cytokine inhibition. J Exp Med 205: 1535-1541, 2008.

4. Lees JR, Golumbek PT, Sim J, Dorsey D and Russell JH: Regional CNS responses to IFN- $\gamma$ determine lesion localization patterns during EAE pathogenesis. J Exp Med 205: 2633-2642, 2008.

5. Qian X, Shen Q, Goderie SK, He W, Capela A, Davis AA and Temple S: Timing of CNS cell generation: A programmed sequence of neuron and glial cell production from isolated murine cortical stem cells. Neuron 28: 69-80, 2000.

6. Sakakibara S and Okano H: Expression of neural RNA-binding proteins in the postnatal CNS: Implications of their roles in neuronal and glial cell development. J Neurosci 17: 8300-8312, 1997.

7. Raff M, Hart I and Lillien L: Glial cell deversification in the rat optic nerve. Cell Differ Dev 27: 172, 1989.

8. Doucette R: Olfactory ensheathing cells: Potential for glial cell transplantation into areas of CNS injury. Histol Histopathol 10: 503-507, 1995.

9. Nishiyama A, Chang A and Trapp BD: $\mathrm{NG}_{2}+$ glial cells: A novel glial cell population in the adult brain. J Neuropathol Exp Neurol 58: 1113-1124, 1999.

10. Furlan R, Bergami A, Cantarella D, Brambilla E, Taniguchi M, Dellabona P, Casorati G and Martino G: Activation of invariant NKT cells by alphaGalCer administration protects mice from MOG35-55-induced EAE: Critical roles for administration route and IFN- $\gamma$. Eur J Immunol 33: 1830-1838, 2003.

11. Steinman L: A brief history of $T_{H} 17$, the first major revision in the $T_{H} 1 / T_{H} 2$ hypothesis of $T$ cell-mediated tissue damage. Nat Med 13: 139-145, 2007. 
12. Charlton B and Lafferty KJ: The Th1/Th2 balance in autoimmunity. Curr Opin Immunol 7: 793-798, 1995.

13. Arican O, Aral M, Sasmaz S and Ciragil P: Serum levels of TNF- $\alpha$, IFN- $\gamma$, IL-6, IL-8, IL-12, IL-17, and IL-18 in patients with active psoriasis and correlation with disease severity. Mediators Inflamm 2005: 273-279, 2005.

14. Hernandez-Pando R and Rook GA: The role of TNF-alpha in T-cell-mediated inflammation depends on the Th1/Th2 cytokine balance. Immunology 82: 591-595, 1994.

15. Saha RN, Liu X and Pahan K: Up-regulation of BDNF in astrocytes by TNF- $\alpha$ : A case for the neuroprotective role of cytokine. J Neuroimmune Pharmacol 1: 212-222, 2006.

16. Fiorentino DF, Zlotnik A, Vieira P, Mosmann TR, Howard M, Moore KW and O'Garra A: IL-10 acts on the antigen-presenting cell to inhibit cytokine production by Th1 cells. J Immunol 146: 3444-3451, 1991.

17. Ouyang W, Rutz S, Crellin NK, Valdez PA and Hymowitz SG: Regulation and functions of the IL-10 family of cytokines in inflammation and disease. Annu Rev Immunol 29: 71-109, 2011.
18. Tagore A, Gonsalkorale WM, Pravica V, Hajeer AH, McMahon R, Whorwell PJ, Sinnott PJ and Hutchinson IV: Interleukin-10 (IL-10) genotypes in inflammatory bowel disease. Tissue Antigens 54: 386-390, 1999.

19. Benveniste EN, Tang LP and Law RM: Differential regulation of astrocyte TNF- $\alpha$ expression by the cytokines TGF- $\beta$, IL- 6 and IL-10. Int J Dev Neurosci 13: 341-349, 1995.

20. Sun D and Wekerle H: Ia-restricted encephalitogenic $\mathrm{T}$ lymphocytes mediating EAE lyse autoantigen-presenting astrocytes. Nature 320: 70-72, 1986.

21. Wang ZW, Yang J, Kong Q, Zhan XX, Lailong M, Li LL, Sun B, Zhang Y, Wang GY, Li LH et al. Curative effect of calcitriol on experimental autoimmune encephalomyelitis and relevant mechanism. J Chin Bio 11: 24, 2011. (In Chinese).

This work is licensed under a Creative Commons Attribution-NonCommercial-NoDerivatives 4.0 International (CC BY-NC-ND 4.0) License. 